\title{
Observation of blue persistent luminescence in amorphous silica annealed by carbon
}

\author{
Yong Fan ${ }^{1}$, Xiaoyang $\mathrm{He}^{1}$, Yuqiang Liu ${ }^{1}$, Donglin $\mathrm{He}^{1}$, Jiachi Zhang $*^{1}$, Pengfei \\ Feng ${ }^{1}$, Zhipeng $\mathrm{Ci} *^{1}$, Zhenyan Zhao ${ }^{1}$, Yuhua Wang ${ }^{1}$ \\ 1, Key Laboratory for Magnetism Magnetic Materials of the Ministry of Education, \\ Lanzhou University, Lanzhou 730000, P. R. China.
}

Corresponding author: zhangjch@1zu.edu.cn; cizhp@lzu.edu.cn

Tel.: +86-931-8912772, Fax: +86-931-8913554

\section{ABSTRACT:}

Intense blue persistent luminescence (PersL) with sufficiently long duration time was firstly observed in $\mathrm{SiO}_{2}$ particles after calcination with carbon. In indicates that the intense PersL of the optimal $\mathrm{SiO}_{2}$ sample calcinated with carbon $(1: 2)$ at $600{ }^{\circ} \mathrm{C}$ can be recorded for about $1 \mathrm{~h}\left(0.32 \mathrm{mcd} / \mathrm{m}^{2}\right)$ and is actually visible for even more than $3 \mathrm{~h}$ by the dark-adapted vision in darkness. It reveals that the defects formatted during the calcination with carbon should be associated with oxygen vacancies and they play very important roles as emitters and traps, contributing to the PersL. The depth of the dominant traps in the optimal $\mathrm{SiO}_{2}$ sample is calculated to be about $0.71 \mathrm{eV}$, which is 
in a suitable range for PersL $(0.5-0.8 \mathrm{eV})$.

KEYWORDS: A: amorphous materials; B: luminescence; B: optical properties; C: X-ray diffraction; D: phosphors.

\section{Introduction}

Persistent luminescence (PersL) is the phenomenon involving energy traps in materials that are filled during an excitation with ultraviolet (UV) or visible light [1-3]. After the end of excitation, the stored energy is gradually released to emitter centers, which emit light within scores of minutes to hours [4]. The PersL materials have been widely used in safety signs, watch dials, decorative objects, energy, life science and so on $[5,6]$.

At present, researchers have been working to develop efficient PersL materials, and most current works are focused on rare-earth materials $[7,8]$. However, the rare-earth raw materials are expensive, and these PersL materials are generally synthesized by solid state method at high temperature $\left(>1000{ }^{\circ} \mathrm{C}\right)$. In addition, some PersL materials are doped with some toxic or heavy metal ions such as $\mathrm{Ta}^{3+}, \mathrm{Pb}^{3+}, \mathrm{Bi}^{3+}, \mathrm{Cr}^{3+}, \mathrm{Cd}^{3+}$ $[9,10]$. Therefore, it is desirable to develop new PersL materials with the advantages of being low cost, non-toxic, with easy fabrication and excellent PersL properties.

According to these requirements, the $\mathrm{SiO}_{2}$ is considered to be a good candidate [11] If we can induce sufficiently long PersL in $\mathrm{SiO}_{2}$, it would be a great breakthrough and can of course promote relative applications. Recently, some groups have observed the 
blue photoluminescence (PL) in amorphous $\mathrm{SiO}_{2}$ and it revealed that the oxygen vacancies created during the annealing process of $\mathrm{SiO}_{2}$ act as emitters [12]. However, to the best of our knowledge, the sufficiently long PersL ( $t>10$ min) in $\mathrm{SiO}_{2}$ has not been reported thus far.

In this work, the $\mathrm{SiO}_{2}$ material was synthesized by a facile sol-gel method [13] and then it was subjected to calcinations with carbon. As a consequence, sufficiently long $\left(t>1 \mathrm{~h}\right.$ ) blue PersL was clearly observed in the obtained $\mathrm{SiO}_{2}$ material. It is, of course, a great breakthrough in the PersL field. The as-synthesized $\mathrm{SiO}_{2}$ sample exhibits the advantages of low cost, non-toxic, easy fabrication and excellent PersL properties. Particularly, the size of $\mathrm{SiO}_{2}$ particles can be easily controlled. Therefore, this $\mathrm{SiO}_{2}$ PersL material is potential to be used to label cells for biological and biomedical applications [2,3]. Moreover, the cheap PersL material can also used as pollution settlement detection powder, and other natural markers.

\section{Experimental procedures}

The $\mathrm{SiO}_{2}$ raw material was synthesized by a traditional sol-gel method. Solution A was prepared with $19 \mathrm{ml}$ of alcohol, $3 \mathrm{ml}$ of water and $3.6 \mathrm{ml}$ of ammonia (28\%). Solution B was prepared with $1.4 \mathrm{ml}$ of tetraethyl orthosilioate (28.0\%) and $3.6 \mathrm{ml}$ of alcohol. Firstly, solution A was dropwise added into solution B. The mixed solution was rocked back and forth to prevent gel formation. Secondly, it was stirred for $2-4 \mathrm{~h}$ until it appeared milky with no layering, and then it rested for $24 \mathrm{~h}$. The solution was shaken for half an hour by a sonic oscillator, cleaned with the alcohol by a high-speed 
centrifugal machine and then dried at $60{ }^{\circ} \mathrm{C}$. Then, the $\mathrm{SiO}_{2}$ samples mixed with different amounts of carbon powders (weight ratio 1:1.5, 1:2, 1:2.5 and 1:3) were subjected to calcinations at different temperatures $\left(450,550,600,650\right.$ and $\left.700{ }^{\circ} \mathrm{C}\right)$ for $6 \mathrm{~h}$ to obtain the final products.

A Rigaku D/Max-2400 X-ray diffractometer was employed to check the phases of all the samples. PL and PersL spectra were obtained by a FLS-920T spectrophotometer with a $450 \mathrm{~W}$ xenon arc lamp (Xe900) as the light source. A PR305 instrument and a FJ-417A TL meter were used to measure the PersL decay curve and thermoluminescence glow curves, respectively. An X-ray photoelectron spectroscopy (XPS) was used for the qualitative analysis of samples. The morphology of the $\mathrm{SiO}_{2}$ particles was obtained by a scanning electron microscope SEM (S-3400 and S-4800).

\section{Results and discussion}

Fig. 1(a) shows the XRD patterns of the $\mathrm{SiO}_{2}$ samples without calcination and with calcination by carbon (weight ratio is $1: 2)$ at different temperatures $(T=450,550,600$, 650 and $700{ }^{\circ} \mathrm{C}$ ). The amorphous nature of $\mathrm{SiO}_{2}$ within the sample is apparent by the broad band in $15-30^{\circ}$ and it indicates that the XRD patterns don't exhibit clear variation for the calcinations [14]. Fig. 1(b) gives the XPS spectra of the typical $\mathrm{SiO}_{2}$ sample after calcination by carbon at $600{ }^{\circ} \mathrm{C}$ and the two major peaks at 103.3 and $532.8 \mathrm{eV}$ correspond to the binding energies of $\mathrm{Si} 2 \mathrm{p}$ and $\mathrm{O} 1 \mathrm{~s}[15,16]$, respectively. It demonstrates that the as-synthesized samples are amorphous $\mathrm{SiO}_{2}$. Fig. 1(c), (d), (e) 
and (f) show the SEM of the typical $\mathrm{SiO}_{2}$ samples without calcination and with calcination at $400{ }^{\circ} \mathrm{C}, 600{ }^{\circ} \mathrm{C}$ and $700{ }^{\circ} \mathrm{C}$, respectively. It clearly illustrates that the $\mathrm{SiO}_{2}$ particles with calcination by carbon at $400{ }^{\circ} \mathrm{C}$ keep the nano-sphere morphology, and the average particle size is about $200-500 \mathrm{~nm}$. However, when the temperature increases to $600{ }^{\circ} \mathrm{C}$, it shows clear agglomeration.

Fig. 2 shows the PL excitation $\left(\lambda_{\mathrm{em}}=438 \mathrm{~nm}\right)$ and emission $\left(\lambda_{\mathrm{ex}}=365 \mathrm{~nm}\right)$ spectra (a) and the PersL emission spectrum (b) of the optimal $\mathrm{SiO}_{2}$ sample after calcination with carbon $(1: 2)$ at $600{ }^{\circ} \mathrm{C}$. The broad excitation band peaked at $365 \mathrm{~nm}$ and the emission band, which peaked at $438 \mathrm{~nm}$, can be observed clearly. The PL mechanism of $\mathrm{SiO}_{2}$ particles is essential different from that of the rare earth doped phosphors and it should be associated with the recombination of $\mathrm{F}$ centers (negative oxygen vacancies) with holes.[17,20] Generally, the $\mathrm{SiO}_{2}$ particles are synthesized by wet chemical methods. It is reasonable to expect that the surfaces of highly hydroxylated silicon, some $\mathrm{Si}$ atoms are bonded to more than two $\mathrm{OH}$ groups. When the de-hydroxylation reaction occurs between the two geminal silanols facing to each other, an edge-sharing dimmer is expected to be formed (scheme 1 in Fig. 2). However, there is another possibility that a pair of $=\mathrm{Si}\left(\mathrm{O}_{2}\right)$ and $=\mathrm{Si}$;, which are called dioxasilirane and siylene, respectively, is created (scheme 2 in Fig. 2). Since the bonding configurations of the products formed from schemes 1 and 2 are rather different from those in the normal siloxane bridges, there configurations can be viewed as defective oxide structures (oxygen vacancies) that might be responsible for the blue PL emission $[17,18]$. Accordingly, as shown in the following reaction (1) and 
(2), the PL progress could be described as follows: under UV irradiation, the electrons $\left(e^{-}\right)$are promoted and are trapped by the $\mathrm{F}^{+}$centers, creating $\mathrm{F}$ centers and holes $\left(h^{+}\right)$. Then, the recombination of $\mathrm{F}$ centers (negative oxygen vacancies) with holes creates the excited states of the emitter, $\left(\mathrm{F}^{+}\right)^{*}$. At last, these excited emitters transfer energy to the ground state giving out the blue light emission.

$$
\begin{aligned}
& e^{-}+\mathrm{F}^{+}+h v_{1}(365 \mathrm{~nm}) \rightarrow \mathrm{F}+h^{+} \\
& \mathrm{F}+h^{+} \rightarrow\left(\mathrm{F}^{+}\right)^{*} \rightarrow \mathrm{F}^{+}+h v_{2}(438 \mathrm{~nm})
\end{aligned}
$$

In additional, as shown in the Fig. 2(d), the CIE coordinations were calculated to be $(0.151,0.093)$ and $(0.149,0.071)$, respectively, and the two points clearly are located in blue region.

Fig. 3 investigates the influences of the calcinations temperature (a, $T=450,550$, 600,650 and $\left.700{ }^{\circ} \mathrm{C}\right)$ and the weight ratio $\left(R_{\mathrm{w}}\right)$ of $\mathrm{SiO}_{2}$ vs. carbon $\left(\mathrm{b}, R_{\mathrm{w}}=1: 1.5,1: 2\right.$, 1:2.5 and 1:3) on the PersL duration time of the $\mathrm{SiO}_{2}$ samples. Fig. 3(c) exhibits the PersL decay curve in a double logic diagram of the $\mathrm{SiO}_{2}$ samples calcinated with carbon $(1: 2)$ at different temperature $\left(T=450,550,600,650\right.$ and $\left.700{ }^{\circ} \mathrm{C}\right)$, and the PersL images of the sample after different delay time are also given in the inset of Fig. 3(c). It was found that the PersL of the optimal sample could be recorded about $3600 \mathrm{~s}$ $\left(0.32 \mathrm{mcd} / \mathrm{m}^{2}\right)$. Since the eye sensitivity is about 100 times better than this value, we can actually observe much longer PersL (actually more than $3 \mathrm{~h}$ ) by using the “dark-adapted eye" in complete darkness.

Moreover, the initial PersL intensity dependant on the calcination temperature has been exhibited in Fig. 3(d) as well. It is found that the PersL intensity is increasing as 
increase of the temperature in reduced atmosphere. The optimal temperature is also $600{ }^{\circ} \mathrm{C}$ and the optimal initial PersL intensity is about $110 \mathrm{mcd} / \mathrm{m}^{2}$. However, when the temperature has increased to $700{ }^{\circ} \mathrm{C}$, both the PersL duration time and intensity have been reduced which is possibly due to the agglomeration of the particles.

Fig. 4(a) shows the TL glow curve of the optimal sample and an asymmetry broad TL band in 300-600 K can be clearly observed. Accordingly, a classical multi-peak fitting method developed by Chen et al [19] has been applied to reveal the trap depth (E) and trap densities $\left(n_{0}\right)$, as presented in Fig. 4(a).

$$
I(T)=s n_{o} \exp \left(-\frac{E}{k T}\right)\left[1+(b-1) \frac{s}{\beta} \times \int_{T_{0}}^{T} \exp \left(-\frac{E}{k T^{\prime}}\right) d T^{\prime}\right]^{-\frac{b}{b-1}}
$$

where $E$ is the trap depth, and $\mathrm{n}_{0}$ is the trap density; $k$ is the Boltzmann constant; $b$ is the kinetics order parameter; $s$ is the frequency factor; and $\beta$ is the heating rate (1 $\mathrm{K} / \mathrm{s}$ for this work). As shown in Fig. 4(a), the TL band consists of three components at about 343, 364 and $409 \mathrm{~K}$ corresponding to the shallow, medium and deep traps, respectively. The accurate attribution of these TL bands remains an open question, but it must be associated with the oxygen vacancies created during calcination in reduced atmosphere. The small errors between the fitting curve and the experimental points are shown in Fig. 4(b), and it indicates that the fitting results shown in Table 1 are reliable. Table 1 shows the fitting parameters of the different TL peaks calcinated at different temperature by carbon, and it can be seen that the density $\left(n_{0}\right)$ of the dominant shallow traps is increasing as increase of the calcination temperatures. It is well known that the calcination at high temperature can create more oxygen vacancies 
in materials [17], and thus it means that the density of traps in the as-synthesized material has been clearly increased for the calcinations at higher temperatures. Since the traps play important roles on PersL, this result can well explain the reason why the PersL changes with the temperatures. Moreover, the initial depths of the dominant shallow traps corresponding to the $343 \mathrm{~K}$ is calculated to the about $0.710 \mathrm{eV}$, which is clearly suitable for PersL.

Fig.4(c) shows the TL curves of the typical $\mathrm{SiO}_{2}$ samples without calcination and with calcination at $450{ }^{\circ} \mathrm{C}$ and $600{ }^{\circ} \mathrm{C}$ in air and by carbon, respectively. It is important to note that higher TL intensity is obtained in the sample with calcination in reduced atmosphere than that with calcination in air. This result clearly indicates that these TL band should be more related to defective oxide structures (oxygen vacancies). Fig. 4(d) exhibits the results of the typical TL fading experiments; the TL curves were measured after irradiation and then different delay times. It indicates that the shallow traps are mainly responsible for the PersL in $3600 \mathrm{~s}$, and the contribution of the deep traps (409 K) can actually be ignored because its TL intensity still keeps 60\%, even after $3600 \mathrm{~s}$.

\section{Conclusions}

Amorphous $\mathrm{SiO}_{2}$ material with sufficiently blue PersL has been successfully obtained by calcination with carbon. The PersL of the optimal $\mathrm{SiO}_{2}$ sample calcinated with carbon $(1: 2)$ at $600{ }^{\circ} \mathrm{C}$ can be recorded for $3600 \mathrm{~s}\left(0.32 \mathrm{mcd} / \mathrm{m}^{2}\right)$, and visibly observed much longer $(3 \mathrm{~h})$ by using a dark-adapted eye in darkness. It reveals that a 
defect pair consisting of a dioxasilirane is associated with the emitter; and the shallow traps $(0.71 \mathrm{eV})$ due to oxygen vacancies created during the calcination with carbon should be mainly responsible for the formation of the PersL.

\section{Acknowledgments}

This work was supported by the National Nature Science Foundation of China (No. 10904057), the Fundamental Research Funds for Central Universities (Nos. Lzjbky-2015-112 and lzujbky-2015-113), and the National Science Foundation for Fostering Talents in Basic Research of the National Natural Science Foundation of China (Nos. 041105 and 041106).

\section{References}

[1] X. Liu, X.X. Zhang, Z. Zhou, Preparation and luminescence properties of patterned $\mathrm{SOG} / \mathrm{Sr}_{2} \mathrm{MgSi}_{2} \mathrm{O}_{7}: \mathrm{Eu}^{2+}, \mathrm{Dy}^{3+}$ long-persistent luminescence composite thin films by one-step photolithography, Mater. Res. Bull. 79 (2016) 84-89.

[2] T. Maldiney, A. Bessiere, J. Seguin, E. Teston, S. Sharma, B. Viana, J. Bos, P. Dorenbos, The in vivo activation of persistent nanophosphors for optical imaging of vascularization, tumours and grafted cells, Nat. Mater. 13 (2014) 418-426.

[3] F. Liu, Y. Liang, Z. Pan, Detection of Up-converted Persistent Luminescence in the Near Infrared Emitted by the $\mathrm{Zn}_{3} \mathrm{Ga}_{2} \mathrm{GeO}_{8}: \mathrm{Cr}^{3+}, \mathrm{Yb}^{3+}, \mathrm{Er}^{3+}$ Phosphor, Phys. Rev. Lett. 113 (2014) 117401.

[4] O. Hai, H.Y. Jiang, D. Xu, M. Li, The effect of grain surface on the long afterglow 
properties of $\mathrm{Sr}_{2} \mathrm{MgSi}_{2} \mathrm{O}_{7}: \mathrm{Eu}^{2+}, \mathrm{Dy}^{3+}$, Mater. Res. Bull. 76 (2016) 358-364.

[5] T.Wang, W.J. Bian, D.C. Zhou, J.B. Qiu, X. Yu, X.H. Xu, Red long lasting phosphorescence in $\mathrm{Ca}_{2} \mathrm{Ge}_{7} \mathrm{O}_{16}: \mathrm{Sm}^{3+}$ via persistent energy transfer from the host to $\mathrm{Sm}^{3+}$. Mater. Res. Bull. 74 (2016) 151-155.

[6] F. Yu, Y. Yang, X. Su, C. Mi, J. H. Seo, Novel long persistent luminescence phosphors: $\mathrm{Yb}^{2+}$ codoped $\mathrm{MAl}_{2} \mathrm{O}_{4}(\mathrm{M}=\mathrm{Ba}, \mathrm{Sr})$. Opt. Mater. Express. 5 (2015) 585.

[7] Y.H. Jin, Y.H. Hu, Different luminescent behaviors between photoluminescence and persistent luminescence in $\mathrm{Tb}^{3+}$ doped $\mathrm{Li}_{2} \mathrm{CaGeO}_{4}$ phosphors. Mater. Res. Bull. $61(2015) 16-21$.

[8] W.Z. Yan, F. Liu, Y.Y. Lu, X.J. Wang, M. Yin, Z.W. Pan, Near infrared long-persistent phosphorescence in $\mathrm{La}_{3} \mathrm{Ga}_{5} \mathrm{GeO}_{14}: \mathrm{Cr}^{3+}$ phosphor, Opt. Express. 18 (2010) 20215-20221 .

[9] Y.H. Mei, H. Xu, J.C. Zhang, Z.P. Ci, M.X. Duan, S.L. Peng, Z.Y. Zhang, W. Tian, Y. Lu, Y.H. Wang, Wang, Design and spectral control of a novel ultraviolet emitting long lasting phosphor for assisting $\mathrm{TiO}_{2}$ photocatalysis: $\mathrm{Zn}_{2} \mathrm{SiO}_{4}: \mathrm{Ga}^{3+}, \mathrm{Bi}^{3+}$, J. Alloys Compd. 622 (2015) 908-912.

[10] P.I. Macfarlane, T.P.J. Han, B. Henderson, A.A. Kaminskii, $\mathrm{Cr}^{3+}$ luminescence in calcium and strontium gallogermanate, Opt. Mater. 3 (1994) 15-24.

[11] Q. Cai, Z.S. Luo, W.Q. Pang, et al, Dilute solution routes to various controllable morphologies of MCM-41 silica with a basic medium, Chem. Mater. 13 (2001) 258-263. 
[12] H.J. Chang, Y.F. Chen, H.P. Lin, Strong visible photoluminescence from $\mathrm{SiO}_{2}$ nanotubes at room temperature, Appl. Phys. Lett. 78 (2001) 3791.

[13] M.A. Fardad, Catalysts and the structure of $\mathrm{SiO}_{2}$ sol-gel films, J. Mater. Sci. 35 (2000) 1835-1841.

[14] C.L. Pang, H. Cui, C.X. Wang, Synthesis and photoluminescence of ultralong amorphous $\mathrm{SiO}_{2}$ nanowires catalysed by germanium, CrystEngComm. 13 (2011) $4082-4085$.

[15] B.S. Zhuo, Y.G. Li, X.K. Zhang, A.C. Yang, Preparation and characterization of amorphous $\mathrm{SiO}_{2}$ nanowires, Mater. Sci. Eng. B 172 (2010) 15-17.

[16] Y.H. Ryu, Y.J. Tak, K. Yong, Direct growth of cor-shell $\mathrm{SiC}_{-} \mathrm{SiO}_{2}$ nanowires and field emission characteristics, Nanotechnology 16 (2005) S370-S374.

[17] Takashi Uchino, Naoko Kurumoto, Natsuko Sagawa, Structure and formation mechanism of blue-light-emitting centers in silicon and silica-based nanostructured materials, Phys. Rev. B 73 (2006) 1098-0121.

[18] T. Uchino, M. Takahashi, and T. Yoko, Phys. Rev. Lett. 86 (2001) 4560-5522 .

[19] R. Chen, Glow curves with general order kinetics, J. Electrochem. Soc. 116 (1969) 1254-1257.

[20] J.C. Zhang, M.H. Yu, Q.S. Qin, H.L. Zhou, M.J. Zhou, X.H. Xu, Y.H. Wang, The persistent luminescence and up conversion photostimulated luminescence properties of nondoped Mg2SnO4 material, J. Appl. Phys. 108 (2010) 123518-123524. 
Table 1 Fitting parameters of the different TL peaks calcinated at different temperature $\left(\mathrm{T}=450,550,600,650\right.$ and $\left.700{ }^{\circ} \mathrm{C}\right)$ by carbon.

\begin{tabular}{|c|c|c|c|c|c|c|}
\hline & \multicolumn{2}{|c|}{ Peak1 } & \multicolumn{2}{|c|}{ Peak2 } & \multicolumn{2}{|c|}{ Peak3 } \\
\hline & $E(\mathrm{ev})$ & $n_{0}\left(\mathrm{~cm}^{-3}\right)$ & $E(\mathrm{ev})$ & $n_{0}\left(\mathrm{~cm}^{-3}\right)$ & $E(\mathrm{ev})$ & $n_{0}\left(\mathrm{~cm}^{-3}\right)$ \\
\hline $450{ }^{\circ} \mathrm{C}$ & 0.890 & $8.882 \times 10^{3}$ & 0.790 & $1.183 \times 10^{4}$ & & 0 \\
\hline $500{ }^{\circ} \mathrm{C}$ & 0.890 & $1.510 \times 10^{4}$ & 0.930 & $2.519 \times 10^{4}$ & & 0 \\
\hline $550{ }^{\circ} \mathrm{C}$ & 0.850 & $2.227 \times 10^{4}$ & 0.930 & $2.783 \times 10^{4}$ & & 0 \\
\hline $600{ }^{\circ} \mathrm{C}$ & 0.710 & $3.132 \times 10^{4}$ & 0.650 & $2.574 \times 10^{4}$ & 0.870 & $7.711 \times 10^{3}$ \\
\hline $700{ }^{\circ} \mathrm{C}$ & & & 0.990 & $6.158 \times 10^{3}$ & 0.900 & $4.981 \times 10^{3}$ \\
\hline
\end{tabular}




\section{Figure captions}

Fig. 1 (a) XRD patterns of the typical $\mathrm{SiO}_{2}$ samples without calcination and with calcinations by carbon at $450,500,550,600,650$ and $700{ }^{\circ} \mathrm{C}$, respectively; (b) XPS of the typical $\mathrm{SiO}_{2}$ sample calcinated at $600{ }^{\circ} \mathrm{C}$; (c), (d), (e) and (f) SEM of the typical $\mathrm{SiO}_{2}$ samples without calcination and with calcinations at $400{ }^{\circ} \mathrm{C}, 600{ }^{\circ} \mathrm{C}$ and $700{ }^{\circ} \mathrm{C}$, respectively.

Fig. 2 (a) PL excitation and emission spectra and (b) PersL spectrum of the typical $\mathrm{SiO} 2$ sample with calcination by carbon $(1: 2)$ at $600{ }^{\circ} \mathrm{C}$; (c) formation of the defect pairs; (d) CIE chromaticity diagram.

Fig. 3 The influences of (a) the calcination temperatures $(T=450,550,600,650$ and $\left.700{ }^{\circ} \mathrm{C}\right)$ and (b) the weight ratio of $\mathrm{SiO}_{2}$ vs. carbon $\left(R_{\mathrm{w}}=1: 1.5,1: 2,1: 2.5\right.$ and 1:3) on the PersL duration time of the $\mathrm{SiO}_{2}$ samples. (c) The PersL decay curves of the $\mathrm{SiO}_{2}$ samples with calcination at different temperatures $\left(T=450,550,600,650\right.$ and $\left.700{ }^{\circ} \mathrm{C}\right)$ by carbon and (inset) the PersL images of the sample at different delay time. (d) The PersL intensities of these samples dependant on temperatures.

Fig. 4 (a) TL curve and (b) fitting errors of the optimal $\mathrm{SiO}_{2}$; (c) TL curves of the $\mathrm{SiO}_{2}$ samples without calcination and with calcination at $450{ }^{\circ} \mathrm{C}$ and $600{ }^{\circ} \mathrm{C}$ in air and by carbon, respectively; (d) results of the TL fading experiments. 

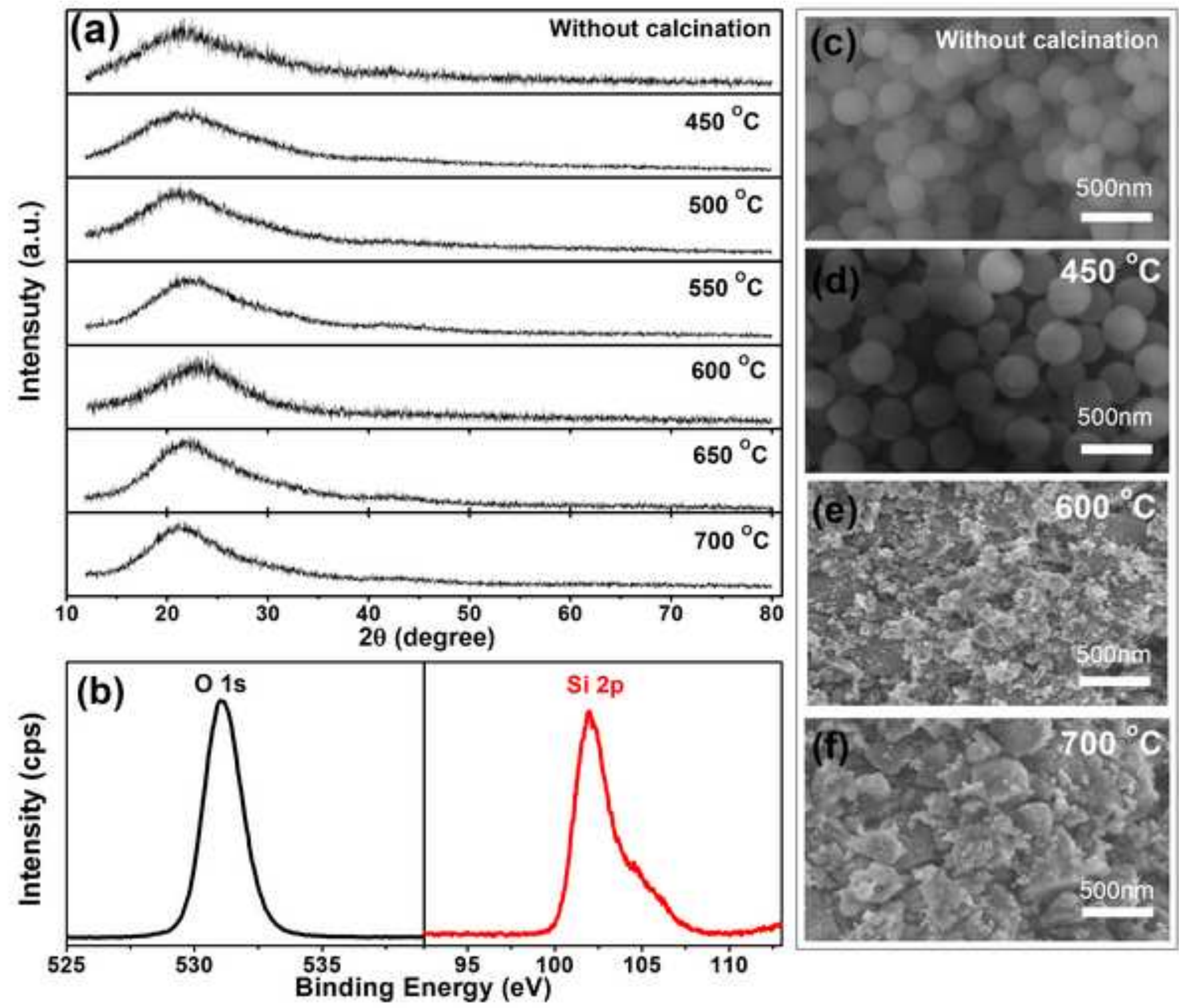

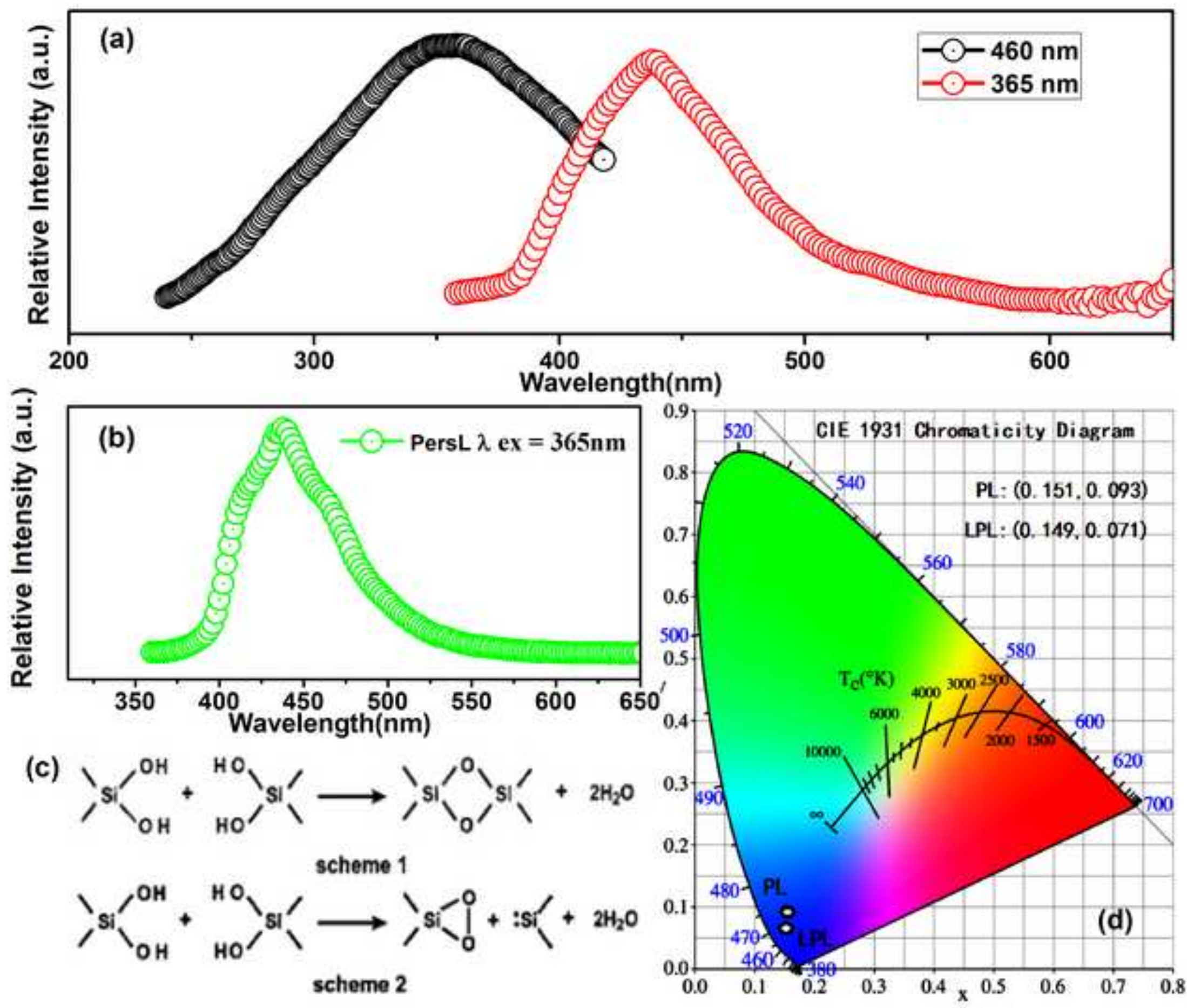

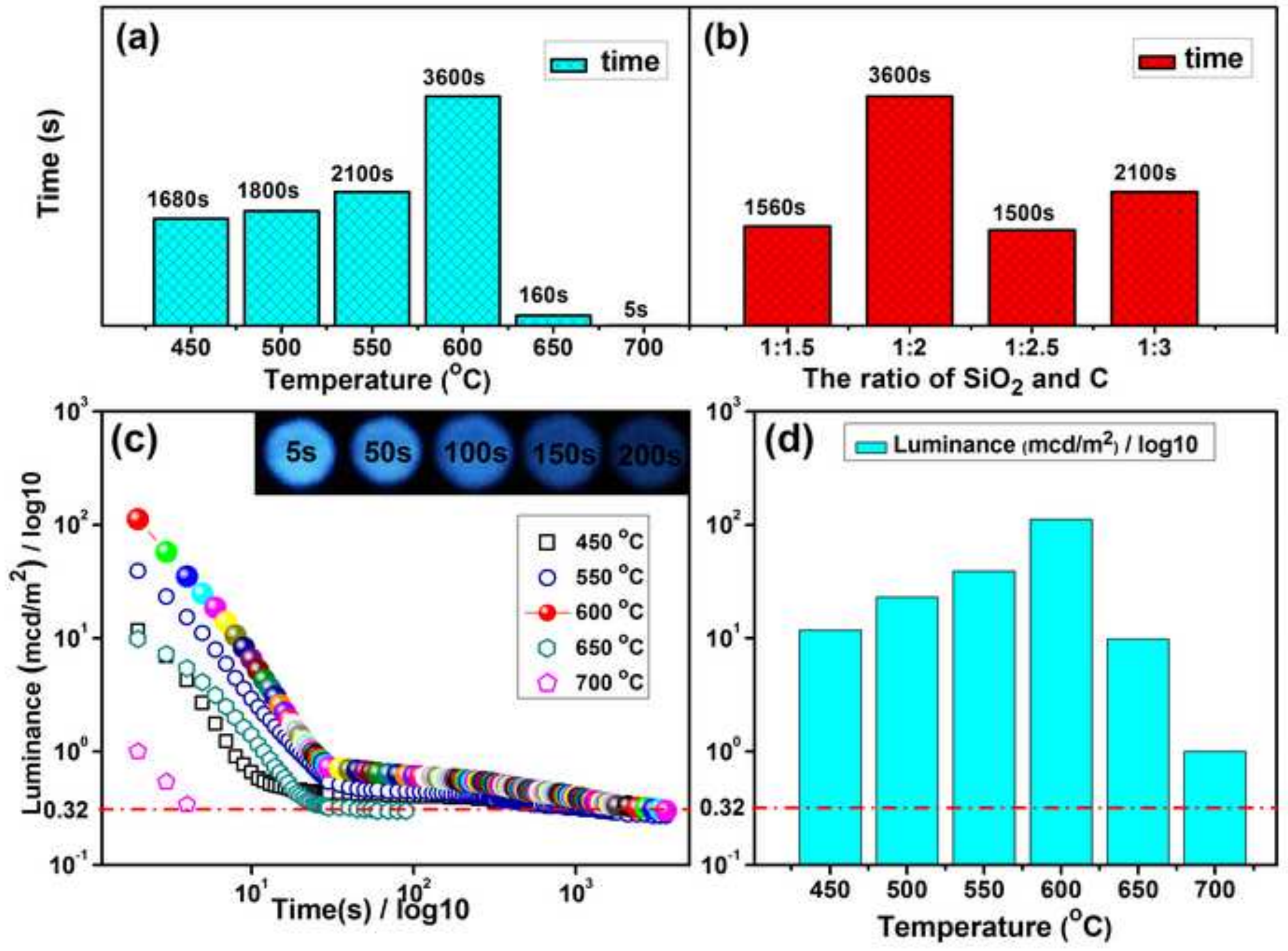

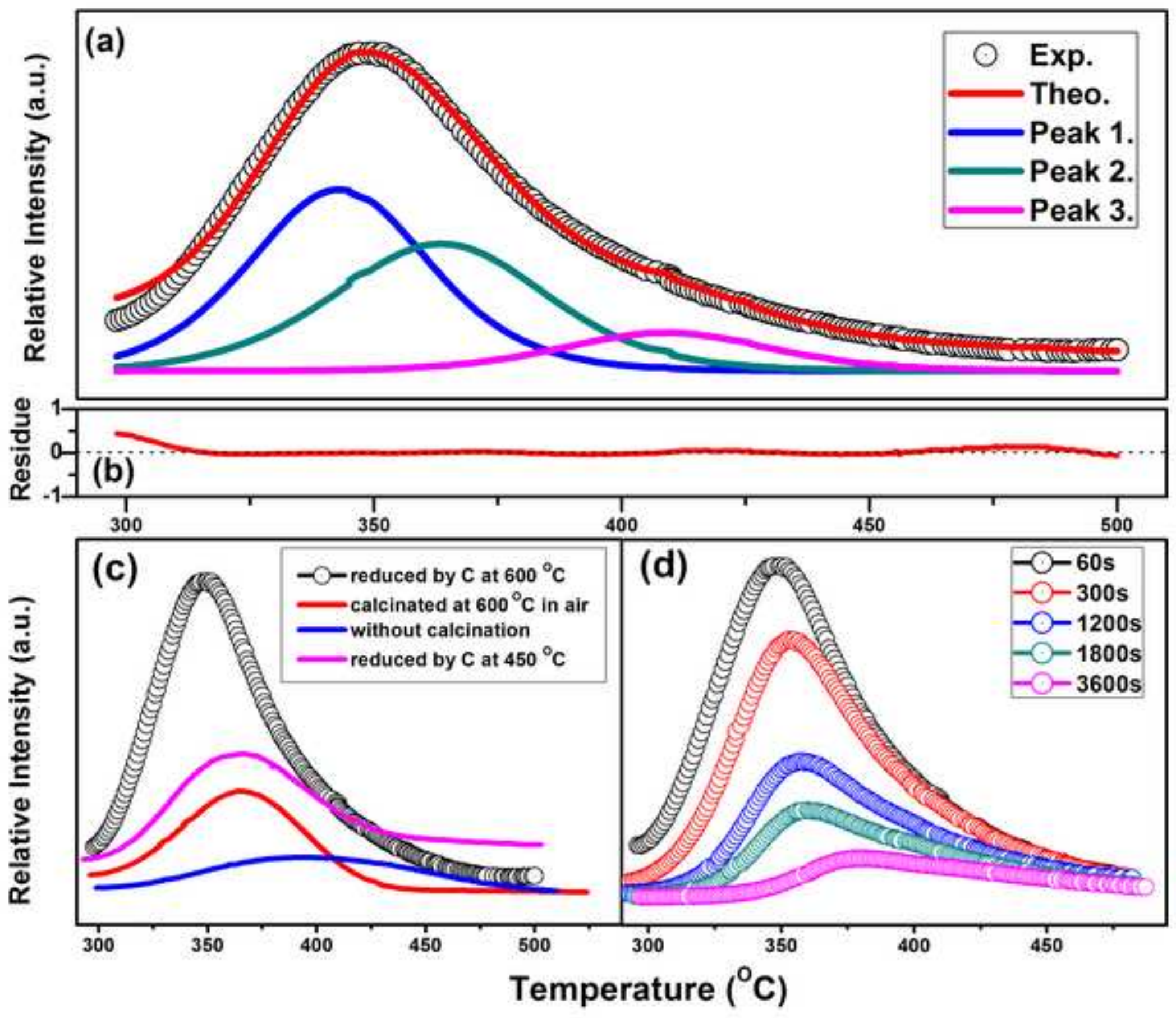
Table 1 Fitting parameters of the different TL peaks calcinated at different temperature $\left(\mathrm{T}=450,550,600,650\right.$ and $\left.700{ }^{\circ} \mathrm{C}\right)$ by carbon.

\begin{tabular}{|c|c|c|c|c|c|c|}
\hline & \multicolumn{2}{|c|}{ Peak1 } & \multicolumn{2}{|c|}{ Peak2 } & \multicolumn{2}{|c|}{ Peak3 } \\
\hline & $E(\mathrm{ev})$ & $n_{0}\left(\mathrm{~cm}^{-3}\right)$ & $E(\mathrm{ev})$ & $n_{0}\left(\mathrm{~cm}^{-3}\right)$ & $E(\mathrm{ev})$ & $n_{0}\left(\mathrm{~cm}^{-3}\right)$ \\
\hline $450{ }^{\circ} \mathrm{C}$ & 0.890 & $8.882 \times 10^{3}$ & 0.790 & $1.183 \times 10^{4}$ & & 0 \\
\hline $500{ }^{\circ} \mathrm{C}$ & 0.890 & $1.510 \times 10^{4}$ & 0.930 & $2.519 \times 10^{4}$ & & 0 \\
\hline $550{ }^{\circ} \mathrm{C}$ & 0.850 & $2.227 \times 10^{4}$ & 0.930 & $2.783 \times 10^{4}$ & & 0 \\
\hline $600{ }^{\circ} \mathrm{C}$ & 0.710 & $3.132 \times 10^{4}$ & 0.650 & $2.574 \times 10^{4}$ & 0.870 & $7.711 \times 10^{3}$ \\
\hline $700{ }^{\circ} \mathrm{C}$ & & & 0.990 & $6.158 \times 10^{3}$ & 0.900 & $4.981 \times 10^{3}$ \\
\hline
\end{tabular}



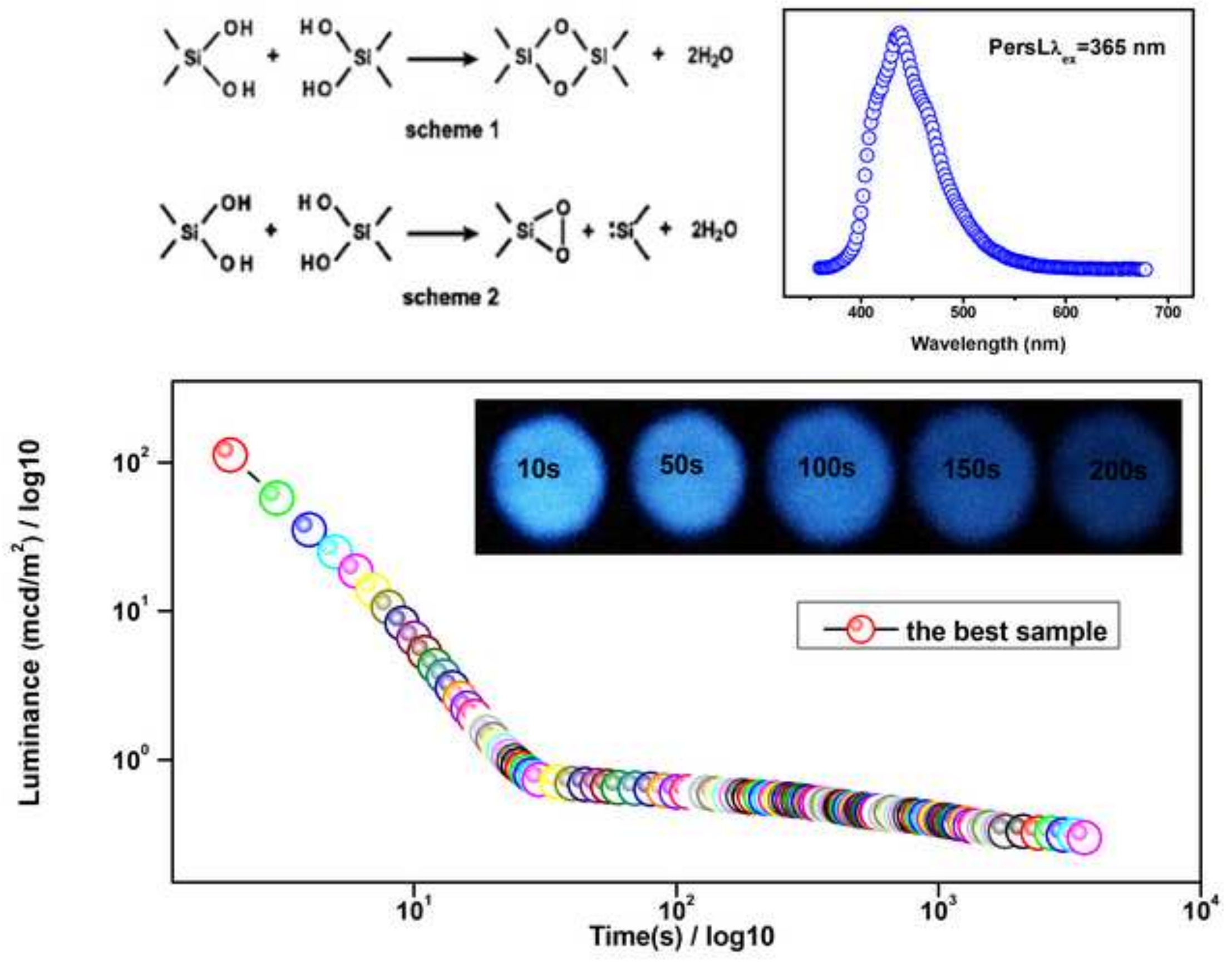